\title{
High power Nd:YVO-KGW conical refraction laser
}

C. Howlader, M. Nadimi, T. Waritanant, K. A. Fedorova, G. S. Sokolovskii, et al.

C. Howlader, M. Nadimi, T. Waritanant, K. A. Fedorova, G. S. Sokolovskii, E. U. Rafailov, A. Major, "High power Nd:YVO-KGW conical refraction laser," Proc. SPIE 10904, Laser Resonators, Microresonators, and Beam Control XXI, 109041X (4 March 2019); doi: 10.1117/12.2508968

SPIE. Event: SPIE LASE, 2019, San Francisco, California, United States 


\title{
High power Nd:YVO-KGW conical refraction laser
}

\author{
C. Howlader ${ }^{1}$, M. Nadimi ${ }^{1}$, T. Waritanant ${ }^{1}$, K.A. Fedorova ${ }^{2}$, G.S. Sokolovskii ${ }^{3,4}$, E.U. Rafailov ${ }^{5,6}$, \\ and A. Major ${ }^{1}$ \\ ${ }^{1}$ Department of Electrical and Computer Engineering, University of Manitoba, Winnipeg, R3T 5V6, \\ Canada \\ ${ }^{2}$ Department of Physics, Philipps-Universität Marburg, Marburg, 35032, Germany \\ ${ }^{3}$ Ioffe Institute, St. Petersburg, 194021, Russia \\ ${ }^{4}$ Saint Petersburg Electrotechnical University (LETI), St. Petersburg, 197022, Russia \\ ${ }^{5}$ School of Engineering \& Applied Science, Aston University, Birmingham, B4 7ET, UK \\ ${ }^{6}$ Saratov State University, Astrakhanskaya Str. 83, Saratov 410012, Russia
}

\begin{abstract}
We have demonstrated the highest conical refraction (CR) laser output power to date by placing a CR crystal inside of a diode-pumped Nd:YVO laser cavity. The CR crystal did not have a significant influence on laser output power as well as efficiency. The CR laser produced the maximum output power of $3.68 \mathrm{~W}$ with the slope efficiency of $42 \%$ and opticalto-optical efficiency of $34 \%$. Therefore, this approach could be an attractive pathway for further power scaling of the CR lasers.
\end{abstract}

Keywords: Conical refraction, solid-state laser, diode-pumped laser, Nd-doped laser crystals

\section{INTRODUCTION}

Conical refraction (CR) is a well-known phenomenon that happens when light propagates along one of the optical axes of a biaxial crystal. As it is shown in Fig. 1, in case of an input beam with Gaussian intensity distribution, CR transforms it into a pair of bright concentric rings separated by a dark Poggendorff ring.

a)

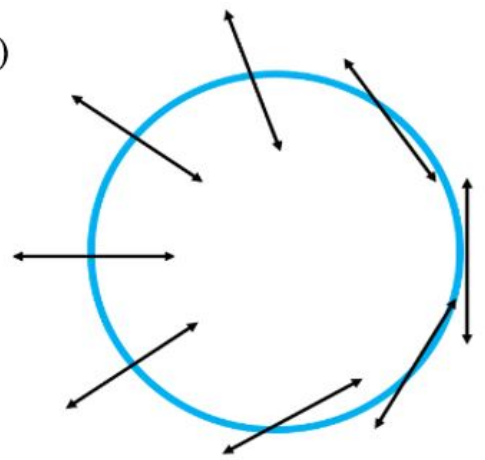

b)

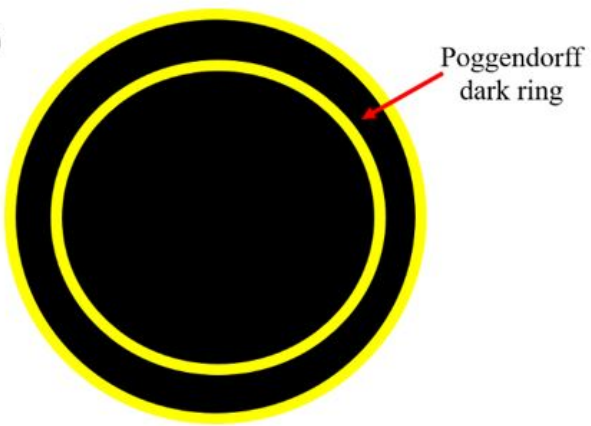

Figure 1. Schematic diagram of the a) polarization along the CR ring pattern and b) Poggendorff dark ring.

In recent years CR has been actively studied due to the potential applications in the fields of optical trapping, free space communication, super-resolution microscopy, cryptography, polarization demultiplexing and multiplexing, polarimetry and mode conversion [1-4]. Currently the most popular way to produce a CR laser output is to combine the CR and optical gain properties of a biaxial Nd:KGW laser crystal [5-10]. However, the output power of the CR Nd:KGW lasers demonstrated to date was limited by moderate thermal conductivity of this crystal [11-14]. To overcome this problem we have demonstrated a passive diode-pumped CR laser using a cascade of an active laser gain medium and a passive CR medium inside of a single optical cavity. 
$\mathrm{Nd}: \mathrm{YVO}_{4}(\mathrm{Nd}: \mathrm{YVO})$ is one of the most popular laser host crystals because of its large stimulated emission cross-section, high absorption coefficient and wide absorption bandwidth at pump wavelength around $810 \mathrm{~nm}$, high laser induced damage threshold, and high slope efficiency. Diode-pumped Nd:YVO ${ }_{4}$ lasers have been demonstrated with multi-watt output and high efficiency in the continuous-wave and pulsed regimes [15-21]. Owing to the natural birefringence and strongly polarized laser radiation, they are a popular choice for efficient process of frequency conversion [22,23] and are widely used to pump Ti:sapphire and Alexandrite lasers [24-27]. The optical and physical properties of Nd:YVO crystal are listed in Table 1 along with the crystals of $\mathrm{Nd}: \mathrm{KGW}$ and $\mathrm{Nd}: \mathrm{YAG}$ for comparison. The emission spectrum of $\mathrm{Nd}: \mathrm{YVO}$ is displayed in Fig. 2.

Table 1. Properties of the Nd:YVO, Nd:YAG and Nd:KGW crystals.

\begin{tabular}{|c|c|c|c|}
\hline Laser crystal type & Nd:YVO4 & Nd:YAG & Nd:KGW \\
\hline Lasing $\lambda \mathrm{nm}$ & $914,1064,1342$ & 946,10641319 & $911,1067.21351$ \\
\hline $\begin{array}{l}\text { Emission cross section, } \mathrm{X} \\
10^{-19} \mathrm{~cm}^{2}\end{array}$ & $25 @ 1064 \mathrm{~nm}$ & $2.8 @ 1064 \mathrm{~nm}$ & $4.3 @ 1067.2 \mathrm{~nm}$ \\
\hline $\begin{array}{l}\text { Fluorescence lifetime }[\mu \mathrm{s}] \\
\text { @doping }\end{array}$ & $90 @ 1 \%$ & 230@1\% & 110@3\% \\
\hline Gain bandwidth [nm] & $0.96 @ 1064 \mathrm{~nm}$ & $0.6 @ 1064 \mathrm{~nm}$ & 2.73 \\
\hline Pump $\lambda$ nm & $808.5,910$ & 807.5 & 811,910 \\
\hline $\begin{array}{l}\text { Absorption coefficient } \\
{\left[\mathrm{cm}^{-1}\right] @ \text { doping } 1 \%}\end{array}$ & $\begin{array}{c}31.4 @ 810 \mathrm{~nm} \\
(\pi, \mathrm{e})\end{array}$ & $7.1 @ 810 \mathrm{~nm}$ & 4.5 \\
\hline $\begin{array}{l}\text { Thermal conductivity } \\
{[\mathrm{W} / \mathrm{mK}]}\end{array}$ & $\begin{array}{l}\| \mathrm{c}: 5.23 \\
\perp \mathrm{c}: 5.10\end{array}$ & 14 & $\begin{array}{l}2.8[100] \\
2.2[010] \\
3.5[001]\end{array}$ \\
\hline
\end{tabular}

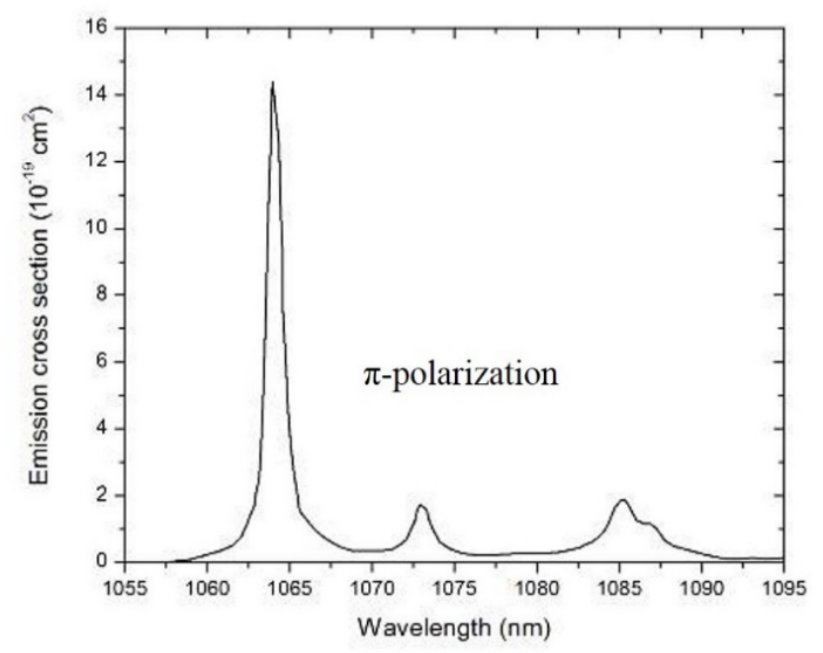

Figure 2. Emission spectrum of $\mathrm{Nd}: \mathrm{YVO}_{4}$ around the main transition at $1064 \mathrm{~nm}$ [28].

As can be seen from Table 1, Nd:YVO has higher thermal conductivity and stimulated emission cross-section both of which can help to produce high output power level from a laser. Taking into account that $1064 \mathrm{~nm}$ is not absorbed by the $\mathrm{KGW}$ crystals, this encouraged us to use $\mathrm{Nd}: \mathrm{YVO}$ as a laser gain medium for passive CR laser in our experiment. 


\section{EXPERIMENTAL SETUP}

The passive CR laser used a Nd:YVO crystal as a laser gain medium. The $20 \mathrm{~mm}$-long 1.5 at.\% Nd:YVO crystal was wrapped with indium foil and water cooled at the flow rate of 1 liter/minute on the top and bottom surfaces in a copper holder at $16{ }^{\circ} \mathrm{C}$. Both surfaces of the crystal were anti-reflection coated at the laser and pump wavelengths. The crystal was pumped by a fiber-coupled diode laser with a fiber core diameter of $110 \mu \mathrm{m}$ and a numerical aperture of 0.22 . Diode pumping used $\sim 914 \mathrm{~nm}$ wavelength which was previously shown to reduce the effect of thermal lensing $[29,30]$ and increase the laser efficiency [31-36].

One arm of the standard z-cavity shown in Fig. 3 contained the laser crystal (Nd:YVO) and the opposite arm contained the CR crystal (KGW) close to an output coupler. The output coupler had 5\% transmission in order to obtain the highest output power and optical efficiency. Cavity design took into account the effect of thermal lensing [37]. The CR crystal was an $18 \mathrm{~mm}$-long anti-reflection coated KGW crystal which was cut along its optical axis. KGW crystals are wellknown for the CR effect [38-42], have good optical quality [11] and are typically used as host for Nd- and $\mathrm{Yb}$-ion lasers [43-53]. A photograph of the developed laser is shown in Fig. 4.

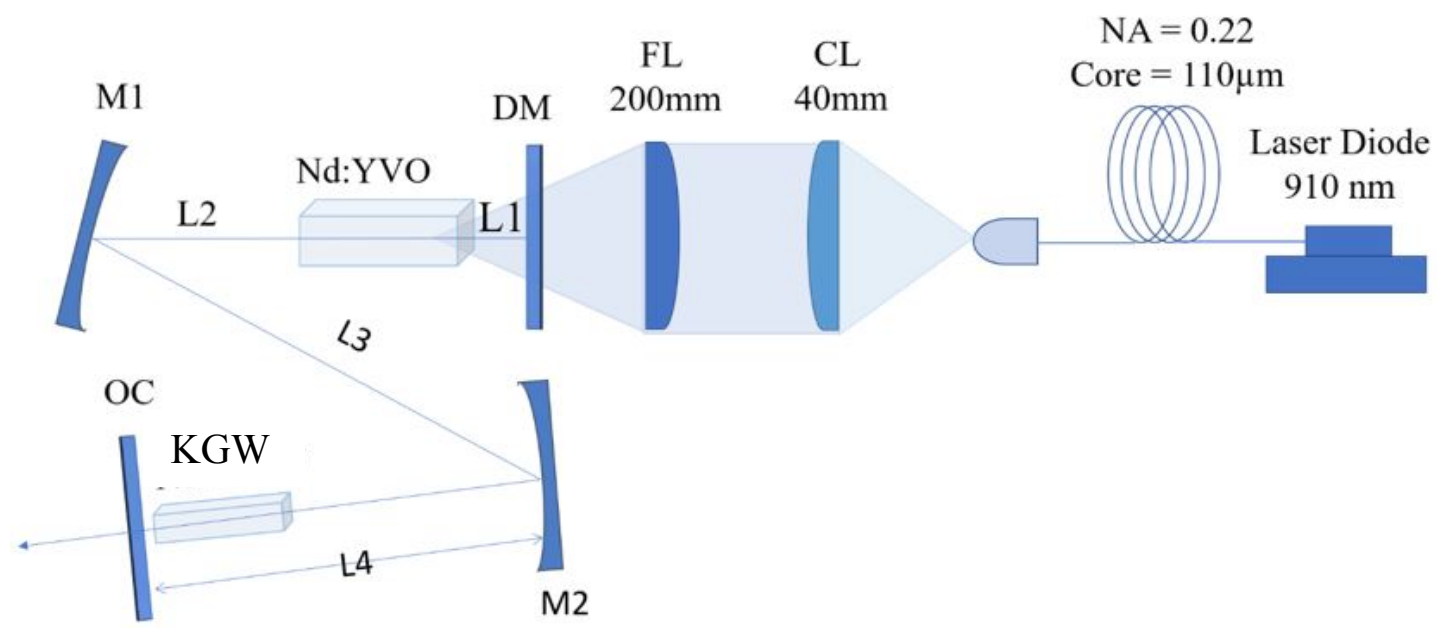

Figure 3. Laser cavity setup of the experimental CR laser. L1, L2, L3 and L4 distances are $24 \mathrm{~mm}, 398 \mathrm{~mm}, 370 \mathrm{~mm}$ and $174 \mathrm{~mm}$, respectively. M1 and M2 are concave mirrors with $\mathrm{r}=500 \mathrm{~mm}$ and $\mathrm{r}=400 \mathrm{~mm}$, respectively. OC is the output coupler and DM is the dichroic pump mirror.

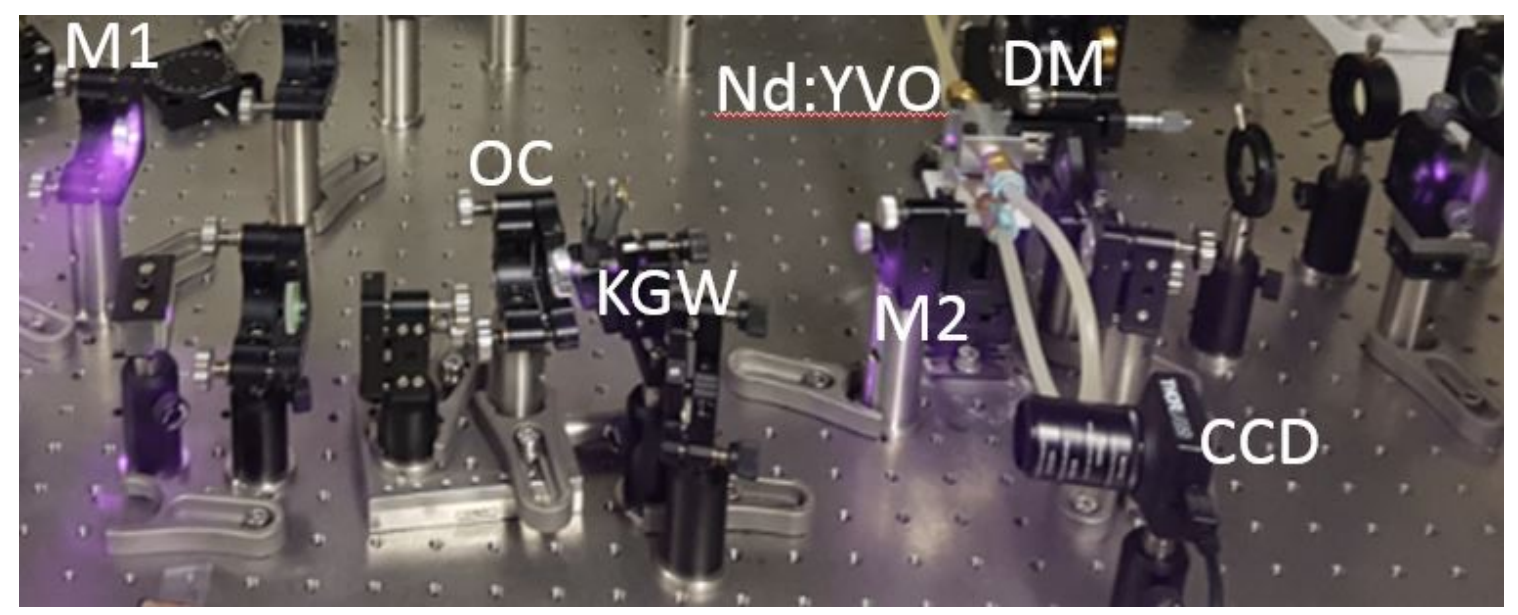

Figure 4. Laser setup on optical table. 
The laser beam diameter was around $550 \mu \mathrm{m}(200 \mu \mathrm{m})$ at the entrance of the Nd:YVO (KGW) crystal. The output beam (at the OC plane) was imaged onto a CCD camera using a single lens with $125 \mathrm{~mm}$ of focal length. Since the theoretical $\mathrm{CR}$ ring radius was $306 \mu \mathrm{m}$ for our crystal, its ratio with the input beam size $\rho_{0}$ was 1.53 . Using a red He-Ne laser the KGW crystal was aligned so that the cavity laser beam could propagate parallel to its optical axis.

\section{RESULTS AND DISCUSSIONS}

The maximum absorbed pump power by the $\mathrm{Nd}$ :YVO crystal was $10.8 \mathrm{~W}$ for the incident pump power of $31.2 \mathrm{~W}$. The laser produced a CR output beam when the KGW crystal was properly oriented. In the CR regime the maximum output power of $3.68 \mathrm{~W}$ was produced. Figure 5 illustrates the output power vs the absorbed pump power. The slope efficiency from a linear fit was $42.3 \%$ and the optical-to-optical efficiency was $34.1 \%$ with respect to the absorbed pump power. To the best of our knowledge, this is the highest CR laser output power demonstrated to date and further power scaling is possible. Previously, up to $3.3 \mathrm{~W}$ were generated from the Nd:KGW CR laser although the beam did not display a CR pattern [6]. Figure 6 displays the optical spectrum of the generated CR laser radiation.

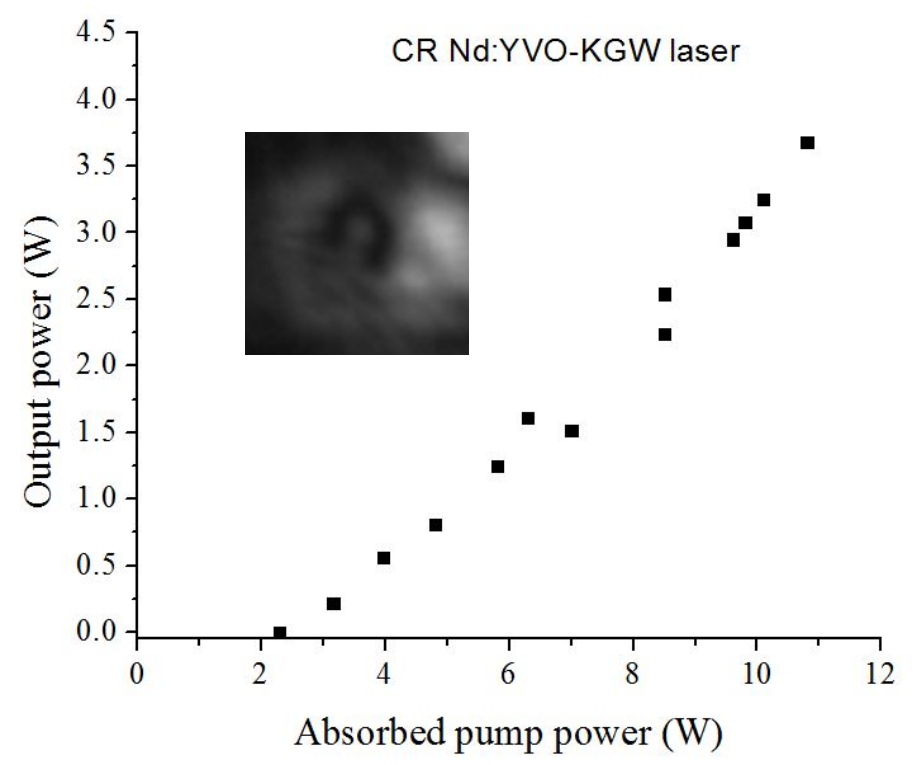

Figure 5. Output power of the CR Nd:YVO-KGW laser. Inset: CR beam intensity profile.

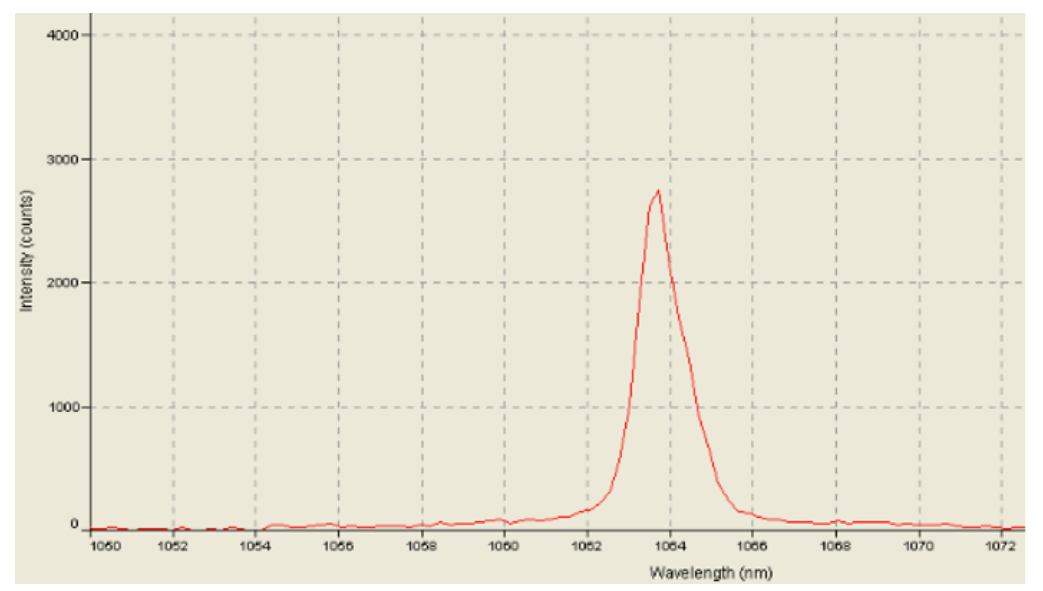

Figure 6. CR laser spectrum. 
The presented results are preliminary. The observed ring shaped profile of the laser radiation is not clear because the $\rho_{0}$ is 1.53. For a clearly resolved double ring CR pattern with a dark Poggendorff ring one should satisfy the condition of the $\rho_{0} \gg>1$ [1]. Nonetheless, we believe that the observed pattern in our experiments can be explained by the conical refraction in the KGW crystal. Further experiments devoted to the accurate characterization of the CR nature of the produced radiation as well as theoretical modeling are in progress.

\section{CONCLUSIONS}

A proof-of-principle experiment to produce a $\mathrm{CR}$ radiation from a laser with the separate gain and $\mathrm{CR}$ media was carried out. The laser used a Nd:YVO crystal as a gain medium and a KGW crystal as a CR medium. The laser was diodepumped and produced up to $3.68 \mathrm{~W}$ of output power at $1064 \mathrm{~nm}$. The initial results indicate that the laser mode focusing conditions should be more tight in order to observe a well behaved CR pattern. At the same time, the proposed approach offers an easy way of achieving this along with the power scaling capabilities.

\section{ACKNOWLEDGEMENTS}

The authors would like to acknowledge funding of this project provided by the Natural Sciences and Engineering Research Council of Canada (NSERC); University of Manitoba (U of M); E.U.R was partially supported by a Grant of Russian Science Foundation (Grant No. 18-15-00172).

\section{REFERENCES}

[1] A. Turpin, Y. V. Loiko, T. K. Kalkandjiev, and J. Mompart, "Conical refraction: fundamentals and applications," Laser Photon. Rev. 10, 750-771 (2016).

[2] D. P. O’Dwyer, K. E. Ballantine, C. F. Phelan, J. G. Lunney, and J. F. Donegan, "Optical trapping using cascade conical refraction of light," Opt. Express 20, 21119-21125 (2012).

[3] C. McDonald, C. McDougall, E. Rafailov, and D. McGloin, "Characterising conical refraction optical tweezers," Opt. Lett. 39, 6691-6694 (2014).

[4] A. Turpin, Y. Loiko, T. K. Kalkandjiev, and J. Mompart, "Free-space optical polarization demultiplexing and multiplexing by means of conical refraction," Opt. Lett. 37, 4197 (2012).

[5] J. Hellström, H. Henricsson, V. Pasiskevicius, U. Bünting, and D. Haussmann, "Polarization-tunable Yb:KGW laser based on internal conical refraction," Opt. Lett. 32, 2783 (2007).

[6] A. Abdolvand, K. G. Wilcox, T. K. Kalkandjiev, and E. U. Rafailov, "Conical refraction Nd:KGd( $\left(\mathrm{WO}_{4}\right)_{2}$ laser," Opt. Express 18, 2753 (2010).

[7] K. G. Wilcox, A. Abdolvand, T. K. Kalkandjiev, and E. U. Rafailov, "Laser with simultaneous Gaussian and conical refraction outputs," Appl. Phys. B 99, 619-622 (2010).

[8] R. Cattoor, I. Manek-Hönninger, D. Rytz, L. Canioni, and M. Eichhorn, "Laser action along and near the optic axis of a holmium-doped $\mathrm{KY}\left(\mathrm{WO}_{4}\right)_{2}$ crystal," Opt. Lett. 39, 6407 (2014).

[9] A. Brenier, "Revealing modes from controlling an off-optical axis conical diffraction laser," Laser Phys. 27, 105001 (2017).

[10] A. Brenier, "Lasing with conical diffraction feature in the $\mathrm{KGd}\left(\mathrm{WO}_{4}\right)_{2}$ : $\mathrm{Nd}$ biaxial crystal," Appl. Phys. B 122, 237 (2016).

[11]I. V. Mochalov, "Laser and nonlinear properties of the potassium gadolinium tungstate laser crystal $\mathrm{KGd}\left(\mathrm{WO}_{4}\right)_{2}: \mathrm{Nd}^{3+}$-(KGW:Nd)," Opt. Eng. 36, 1660 (1997).

[12] P. Loiko, S. Manjooran, K. Yumashev, and A. Major, "Polarization anisotropy of thermal lens in $\mathrm{Yb}: \mathrm{KY}\left(\mathrm{WO}_{4}\right)_{2}$ laser crystal under high-power diode pumping," Appl. Opt. 56, 2937-2945 (2017).

[13] H. Zhao, A. Major, "Orthogonally polarized dual-wavelength Yb:KGW laser induced by thermal lensing," Appl. Phys. B 122(6), 163-169 (2016).

[14]H. Zhao, A. Major, "A continuous wave Yb:KGW laser with polarization-independent pump absorption," Laser Phys. 23, 095001 (2013).

[15]L. McDonagh, R. Wallenstein, R. Knappe, and A. Nebel, "High-efficiency $60 \mathrm{~W}$ TEM 00 Nd:YVO $\mathrm{Yscillator}$ pumped at $888 \mathrm{~nm}$," Opt. Lett. 31(22), 3297-3299 (2006). 
[16] L. McDonagh, R. Wallenstein, and A. Nebel, "111 W, $110 \mathrm{MHz}$ repetition-rate, passively mode-locked TEM 00 $\mathrm{Nd}: \mathrm{YVO}_{4}$ master oscillator power amplifier pumped at $888 \mathrm{~nm}$," Opt. Lett. 32(10), 1259-1261 (2007).

[17] D. Sangla, M. Castaing, F. Balembois, and P. Georges, "Highly efficient Nd:YVO ${ }_{4}$ laser by direct in-band diode pumping at $914 \mathrm{~nm}$, , Opt. Lett. 34(14), 2159-2161 (2009).

[18] T. Waritanant and A. Major, "High efficiency passively mode-locked $\mathrm{Nd}: \mathrm{YVO}_{4}$ laser with direct in-band pumping at 914 nm," Opt. Express 24(12), 12851-12855 (2016).

[19] T. Waritanant and A. Major, "Diode-pumped Nd: $\mathrm{YVO}_{4}$ laser with discrete multi-wavelength tunability and high efficiency," Opt. Lett. 42(6), 1149-1152 (2017).

[20] M. Nadimi, T. Waritanant, and A. Major, "High power and beam quality continuous-wave Nd:GdVO 4 laser inband diode-pumped at $912 \mathrm{~nm}$, " Photon. Res. 5(4), 346-349 (2017).

[21] M. Nadimi, T. Waritanant, and A. Major, "Passively mode-locked high power Nd:GdVO 4 laser with direct inband pumping at $912 \mathrm{~nm}, "$ Laser Phys. Lett. 15(1), 015001 (2018).

[22] A. Major, K. Sukhoy, H. Zhao, and I. T. Lima Jr., "Green sub-nanosecond microchip laser based on BiBO crystals," Laser Phys. 21(1), 57-60 (2011).

[23] H. Zhao, K. Sukhoy, I. T. Lima Jr., and A. Major, "Generation of green second harmonic with $60 \%$ conversion efficiency from a Q-switched microchip laser in MgO:PPLN crystal," Laser Phys. Lett. 9, 355-358 (2012).

[24] S. Ghanbari and A. Major, "High power continuous-wave Alexandrite laser with green pump," Laser Phys. 26(7), 075001 (2016).

[25] S. Ghanbari and A. Major, "High power continuous-wave dual-wavelength Alexandrite laser," Laser Phys. Lett. 14(10), 105001 (2017).

[26] S. Ghanbari, R. Akbari and A. Major, "Femtosecond Kerr-lens mode-locked Alexandrite laser," Opt. Express 24(13), 14836-14840 (2016).

[27] S. Ghanbari, K.A. Fedorova, A.B. Krysa, E.U. Rafailov, and A. Major, "Femtosecond Alexandrite laser passively mode-locked by an InP/InGaP quantum-dot saturable absorber," Opt. Lett. 43(2), 232-234 (2018).

[28] G. Shayeganrad and L. Mashhadi, "Dual-wavelength CW diode-end-pumped a-cut Nd:YVO $\mathrm{YV}_{4}$ laser at 1064.5 and 1085.5 nm," Appl. Phys. B 111(2), 189-194 (2013).

[29] T. Waritanant and A. Major, "Thermal lensing in Nd: $\mathrm{YVO}_{4}$ laser with in-band pumping at 914 nm," Appl. Phys. B 122(5), 135 (2016).

[30] M. Nadimi, T. Waritanant, and A. Major, "Thermal lensing in $\mathrm{Nd}: \mathrm{GdVO}_{4}$ laser with direct in-band pumping at 912 nm," Appl. Phys. B, 124(8), 170 (2018).

[31] T. Waritanant and A. Major, "Discretely selectable multiwavelength operation of a semiconductor saturable absorber mirror mode-locked Nd:YVO 4 laser," Opt. Lett. 42(17), 3331-3334 (2017).

[32] M. Nadimi, A. Major, "Continuous-wave dual-wavelength operation of a diode-pumped Nd:GdVO ${ }_{4}$ laser at the $1063 \& 1071 \mathrm{~nm}, 1063$ \& $1083 \mathrm{~nm}$ and 1083 \& $1086 \mathrm{~nm}$ wavelength pairs," Laser Phys. 28(9), 095001 (2018).

[33] T. Waritanant and A. Major, "Dual-wavelength operation of a diode-pumped $\mathrm{Nd}: \mathrm{YVO}_{4}$ laser at the 1064.1 \& $1073.1 \mathrm{~nm}$ and $1064.1 \& 1085.3 \mathrm{~nm}$ wavelength pairs," Appl. Phys. B 124(5), 87 (2018).

[34] R. C. Talukder, Md. Z. E. Halim, T. Waritanant, and A. Major, "Multiwatt continuous wave Nd:KGW laser with hot-band diode pumping," Opt. Lett. 41(16), 3810-3812 (2016).

[35] Md. Z. E. Halim, R.C. Talukder, T. Waritanant, and A. Major, "Passive mode-locking of a Nd:KGW laser with hot band diode pumping," Laser Phys. Lett. 13(10), 105003 (2016).

[36] M. Nadimi, T. Waritanant, and A. Major, "Discrete multi-wavelength tuning of a continuous wave diodepumped Nd:GdVO 4 laser," Laser Phys. Lett. 15(5), 055002 (2018).

[37]H. Mirzaeian, S. Manjooran, and A. Major, "A simple technique for accurate characterization of thermal lens in solid state lasers," Proc. SPIE 9288, 928802 (2014).

[38] T. K. Kalkandjiev and M. A. Bursukova, "Conical refraction: an experimental introduction," Proc. SPIE 6994, 69940B (2008).

[39] Y. V. Loiko, G. S. Sokolovskii, D. Carnegie, A. Turpin, J. Mompart, and E. U. Rafailov, "Laser beams with conical refraction patterns," Proc. SPIE 8960, 89601Q (2014).

[40] G. S. Sokolovskii, D. J. Carnegie, T. K. Kalkandjiev, and E. U. Rafailov, "Conical Refraction: New observations and a dual cone model," Opt. Express 21, 11125 (2013).

[41] A. Turpin, Y. V. Loiko, T. K. Kalkandkiev, H. Tomizawa, and J. Mompart, "Super-Gaussian conical refraction beam," Opt. Lett. 39, 4349 (2014).

[42] G. S. Sokolovskii, V. Y. Mylnikov, S. N. Losev, K. A. Fedorova, and E. U. Rafailov, "Conical refraction of a high-M2 laser beam,” Proc. SPIE 10090, 100901R (2017). 
[43] A. Major, N. Langford, T. Graf and A. I. Ferguson, "Additive-pulse mode locking of a diode-pumped $\mathrm{Nd}: \mathrm{KGd}\left(\mathrm{WO}_{4}\right)_{2}$ laser,” Appl. Phys. B 75(4-5), 467-469 (2002).

[44]R. Akbari, A. Major, "High-power diode-pumped Kerr-lens mode-locked bulk Yb:KGW laser," Appl. Opt. 56(31), 8838-8844 (2017).

[45] A. Major, L. Giniunas, N. Langford, A.I. Ferguson, D. Burns, E. Bente, and R. Danielius, "Saturable Bragg reflector-based continuous-wave mode locking of $\mathrm{Yb}: \mathrm{KGd}\left(\mathrm{WO}_{4}\right)_{2}$ laser," J. Mod. Opt. 49(5), 787-793 (2002).

[46] R. Akbari and A. Major, "Kerr-lens mode locking of a diode-pumped Yb:KGW laser using an additional intracavity Kerr medium," Laser Phys. Lett. 15(8), 085001 (2018).

[47] H. Zhao and A. Major, "Megawatt peak power level sub-100 fs Yb:KGW oscillators," Opt. Express 22(25), 30425-30431 (2014).

[48] R. Akbari, K. A. Fedorova, E. U. Rafailov, A. Major, "Diode-pumped ultrafast Yb:KGW laser with 56 fs pulses and multi-100 kW peak power based on SESAM and Kerr-lens mode locking," Appl. Phys. B 123(4), 123 (2017).

[49] R. Akbari, H. Zhao, K. A. Fedorova, E. U. Rafailov, and A. Major, "Quantum-dot saturable absorber and Kerrlens mode-locked Yb:KGW laser with $>450 \mathrm{~kW}$ of peak power," Opt. Lett. 41(16), 3771-3774 (2016).

[50] H. Zhao, A. Major, "Dynamic characterization of intracavity losses in broadband quasi-three-level lasers," Opt. Express 22, 26651-26658 (2014).

[51] A. Major, R. Cisek and V. Barzda, "Development of diode-pumped high average power continuous-wave and ultrashort pulse Yb:KGW lasers for nonlinear microscopy," Proc. SPIE 6108, 61080Y (2006).

[52] R. Akbari, H. Zhao, and A. Major, "High-power continuous-wave dual-wavelength operation of a diode-pumped Yb:KGW laser," Opt. Lett. 41(7), 1601-1604 (2016).

[53] A. Major, D. Sandkuijl, V. Barzda, "A diode-pumped continuous-wave Yb:KGW laser with Ng-axis polarized output,” Laser Phys. Lett. 6(11), 779-781 (2009). 